\title{
A Performance Analysis of IEEE 802.11 Networks in the Presence of Hidden Stations
}

\author{
Marek Natkaniec, Andrzej R. Pach \\ University of Mining and Metallurgy, Department of Telecommunications, Cracow, Poland \\ natkanie@kt.agh.edu.pl, pach@kt.agh.edu.pl
}

Key words: $\quad$ Wireless LAN, IEEE 802.11, Hidden Stations, Performance Analysis

Abstract: $\quad$ IEEE 802.11 is a wireless network standard that was completed in 1997. Unfortunately, the medium access protocol described in the standard meets some problems that arise from the presence of so-called hidden stations. This situation can cause degradation of the network performance. The paper describes a simulation analysis of influence of hidden stations on the IEEE 802.11 network efficiency in four different hidden terminals scenarios. The throughput and the mean packet delay as a function of the offered load has been studied. The presented results allow us to determine the usefulness of RTS/CTS mechanism usage in the presence of hidden stations.

\section{INTRODUCTION}

We can observe a permanent growth of interest in the area of WLANs (Wireless Local Area Networks) in last years. WLANs assure easy and free access to existing network infrastructures: LAN, MAN and WAN. These networks can also be attractive for new users by assuring wireless access to databases in magazines, stores, hospitals, airfields, museums etc. IEEE 802.11 [4] is a new wireless network standard that was completed in 1997. There are some vendors of IEEE 802.11 network cards.

The most common medium access algorithm used in WLANs is CSMA (Carrier Sense Multiple Access). In CSMA, every contending station senses the carrier before the transmission. Carrier sense allows avoiding the 
collisions by testing the signal energy in the occupied band. WLANs use a mutation of that scheme called CSMA/CA (Carrier Sense Multiple Access with Collision Avoidance). This algorithm has been employed by the DCF (Distributed Coordination Function) function of IEEE 802.11.

The basic idea of operation of protocols based on CSMA relies on packet transmission avoidance by the station if it detects that the radio channel is busy. Unfortunately, this class of protocols meets some problems that arise from the presence of hidden stations. The situation when some stations do not hear each other happens very often in WLANs. The station sending a data packet cannot be sure whether the packet reaches the destination without collision. This can happen, for example, when a station is not able to hear some other transmissions directed to the same receiver (some stations can operate at geographically separated areas). This situation can cause a substantial degradation of the network performance. The hidden, exposed and intruding station problems are described in details in the next section.

The first protocol that limits an unprofitable hidden stations influence in a single channel has been proposed in [5]. It was called MACA (Multiple Access Collision Avoidance). This protocol use two-way handshaking between the source and destination station. The source station transmits the RTS (Request To Send) packet to the destination station. If it receives the RTS packet correctly, then immediately starts to transmit the mini-packet called CTS (Clear To Send). The proper reception of the CTS packet means that the medium was reserved and the source station can start to transmit its data. There are many other protocols based on RTS/CTS exchange before the data transmission that operates in a single channel [2], [3], [4]. The DFWMAC protocol that realizes the DCF function of the IEEE 802.11 standard belongs to this group.

An influence of hidden stations on fairness of operation and performance of an IEEE 802.11 network has intensively been studied in the literature. An analysis of RTS/CTS usage in DFWMAC protocol was presented in [10]. This work presents the simulation results of a network consisted of eight stations with three hidden ones. It shows the positive effect of RTS/CTS usage. An analysis of fairness of a network consisted of 25 stations with four hidden ones was described in [11]. This work evaluates the network efficiency while exchanging different traffic types generated by different applications with and without usage of RTS/CTS mechanism.

This paper describes a simulation analysis of hidden station influence on the IEEE 802.11 network efficiency for four different hidden terminals scenarios. The throughput (overall and obtained for every station) and the mean packet delay as a function of the offered load has been studied. The presented results allow us to determine the usefulness of RTS/CTS mechanism usage in the presence of hidden stations. 


\section{HIDDEN, EXPOSED AND INTRUDING STATIONS}

The limited area of transmission is characteristic for station operation in a wireless environment. It means that packets transmitted over wireless medium can be received by the stations which are located in the coverage area of the sender. The situation where all stations hear each other occurs very rarely. It brings the problem of hidden stations. A very similar problem arises in the case of exposed stations, that is described below. The presence of any of the mentioned cases brings a serious problem concerned with fair access. It causes the degradation of the network performance. There are four cases of hidden and exposed stations: a hidden sender, a hidden receiver, an exposed sender and an exposed receiver [1], [2].

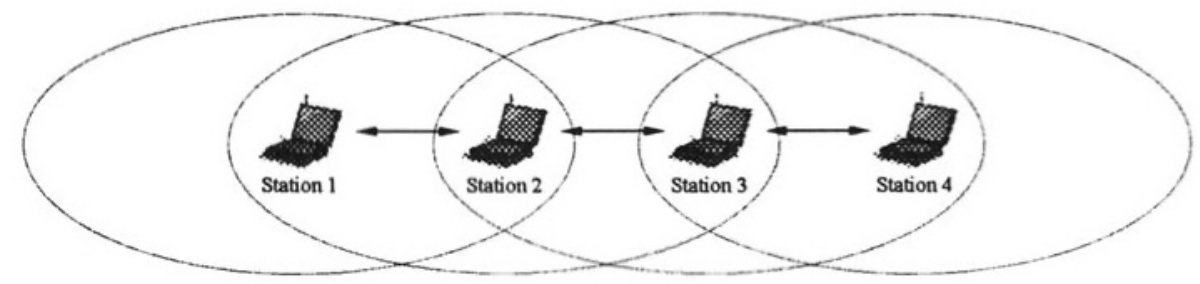

Fig. 1. En example of hidden and exposed stations

Fig. 1 presents four stations that are located in such a way that each station can hear the transmission from the immediate neighbors. When Station 1 transmits a packet to Station 2, Station 3 does not hears the transmission form Station 1. It could happen that during this time Station 3 transmits a packet, so the collision occurs. The Station 2 hears the collision but Station 1 does not. In this situation, when Station 1 transmits to Station 2, Station 3 should defer its transmission. This is the problem of hidden sender. The Station 2 should notify Station 3 about the transmission because Station 1 is not able to do it. Then, every data packet should be preceded by a mini packet handshake. A station should defer its transmission when in response it hears the handshake mini packet. From the above, it follows that Station 3 should defer transmission up to the end of data transmission from Station 1 to Station 2.

The next case is the situation where Station 4 wants to transmit a data packet to Station 3. At first, it sends the control mini packet to Station 3. Station 3 cannot send any packet because it defers. Station 4 will not hear a response then try to retransmit the control packet in order to establish the handshake. This is the case of a hidden receiver. 
The case of an exposed station occurs when the station is within the range of the transmitter and out of range of the receiver. The problem of an exposed sender appears when the exposed station cannot start its own transmission during any other one because it cannot hear the response of a mini packet in the handshake. Let us consider again the case presented in Fig. 1. Station 3 transmits the packet to Station 4. The Station 2 cannot start its transmission to Station 1 because this can cause the collision of packets at Stations 2 and 3.

The exposed receiver problem can be explained as follows. Station 3 transmits a packet to Station 4 . If Station 1 transmits a control mini packet to Station 2, Station 2 is not able to understand any transmission because of collision with transmission from Station 3.

It could also be a situation where the problem of an intruding terminal occurs. This is concerned with mobility of stations. When a terminal moves into the communication range of an occupied receiver, any transmission of the intruding station will cause the collision with any ongoing transmission. The protocols based on single channel environment are endangering to the intruding terminal problem. An unprofitable influence of intruding station can be reduced with the aid of multichannels protocols [3], [7].

\section{OPERATION OF RTS/CTS MECHANISM}

The IEEE 802.11 standard supports two access methods: a mandatory Distributed Coordination Function (DCF) method which is available in both ad hoc and infrastructure configurations, and an optional Point-Coordinated Function (PCF) which is available in certain infrastructure environments and can provide time-bounded services [6].

DCF is the fundamental access method used to support asynchronous data transfer on the best effort basis. All the stations must support DCF. DCF employs the carrier sensing (CS) mechanism. In order to minimize the probability of collisions a random backoff mechanism is used to randomize moments at which medium is tried to be accessed.

The DCF protocol is enhanced further by provision of a virtual CS indication called Net Allocation Vector (NAV) which is based on duration information transferred in special RTS/CTS frames before the data exchange. It allows stations to avoid transmission in time intervals in which the medium is surely busy. The detailed DCF description can be found in [4].

The handshaking usage allows increasing the network performance. The collisions of short information packets and reduction of an unprofitable hidden stations influence can increase the throughput. 


\section{RESULTS OF SIMULATION}

The carried out simulations allows us to determine the realized throughput and the mean packet delay versus the offered load for each station while transmitting 1000 octets data frames for four different hidden station scenarios that are depicted in Fig. 2. The RTS/CTS mechanism was always enabled for all the scenarios in the first part of the study. The possibility of handshaking was disabled for Scenario C and D in second part of our study. The dependencies between the overall throughput and the mean packet delay (for all stations) versus the offered load for all considered scenarios are presented in figures in the following.

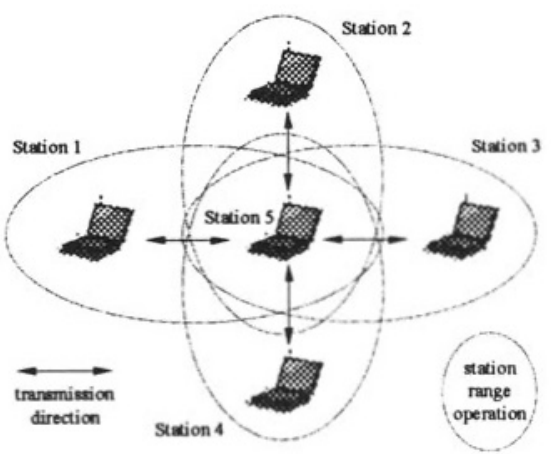

Scenario A

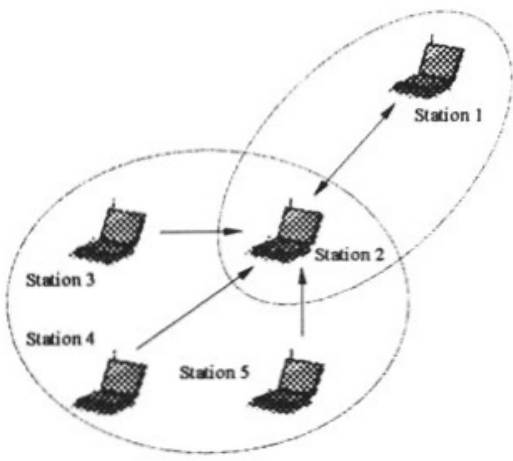

Scenario C

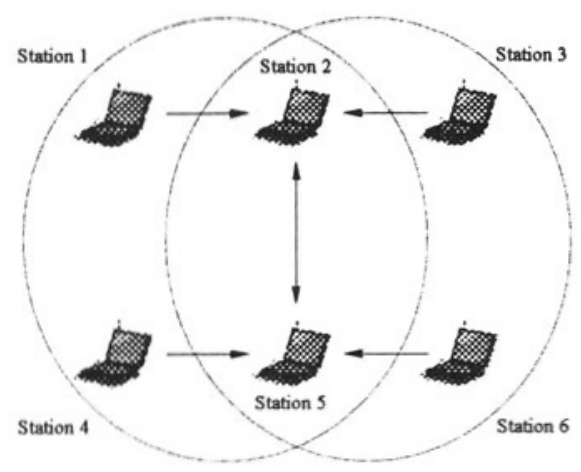

Scenario B

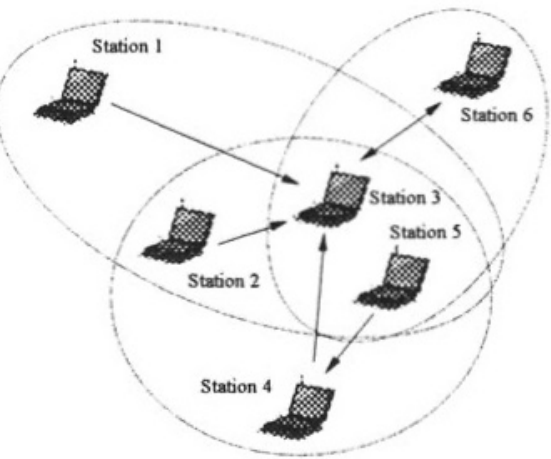

Scenario D

Fig. 2. Four examples of hidden station scenarios

The packet arrivals were realized according to the Poisson distribution. Several assumptions were made to reduce the complexity of the simulation model: 
- The channel is error-free that means that each packet that is transmitted by the sender is successfully received by the receiver.

- The effects of propagation delay are neglected. This is very realistic assumption if the distances are of tens meters between stations.

- There is no station operating in the "power-saving" mode. Each station is "awake" all the time. Then transmitted frames can be received immediately by the destination stations.

The RTS/CTS/DATA/ACK or DATA/ACK mode of transmission was assumed. The network was configured to 2 Mbps medium capacity. Almost all parameters were taken from the standard specification and are adequate to the FHSS (Frequency Hopping Spread Spectrum) physical layer specification. The parameters used throughout all simulations are displayed in Table 1.

Tablel Parameters used throughout all simulations.

\begin{tabular}{llll}
\hline Parameter & Value & Parameter & Value \\
\hline SIFS & $28 \mu \mathrm{s}$ & Minimum number of slots - CWmin & 32 slots \\
DIFS & $130 \mu \mathrm{s}$ & Maximum number of slots - CWmax & 1024 slots \\
Length of RTS & 20 octets & Physical layer preamble & 18 octets \\
Length of CTS & 14 octets & Medium capacity & 2 Mbps \\
Length of ACK & 14 octets & Length of DATA frames & 1000 octets \\
DATA header & 32 octets & Number of retransmissions of RTS frames & 4 \\
Slot time & $50 \mu \mathrm{s}$ & Number of retransmissions of DATA frames & 4 \\
T1 timer & $300 \mu \mathrm{s}$ & Number of hidden stations & variable \\
T3 timer & $300 \mu \mathrm{s}$ & Number of stations & variable \\
Buffer size & 10 frames & RTS_Threshold & RTS/CTS - \\
& & & enabled or \\
& & & disabled \\
\hline
\end{tabular}

The results of simulations are presented in a number of plots in the following:

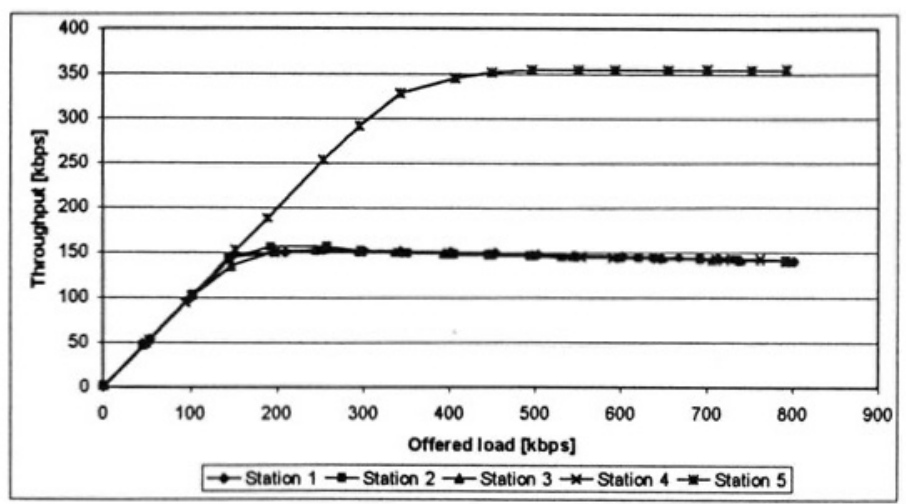

Fig. 3. Throughput versus offered load for Scenario A, RTS/CTS mechanism always enabled 


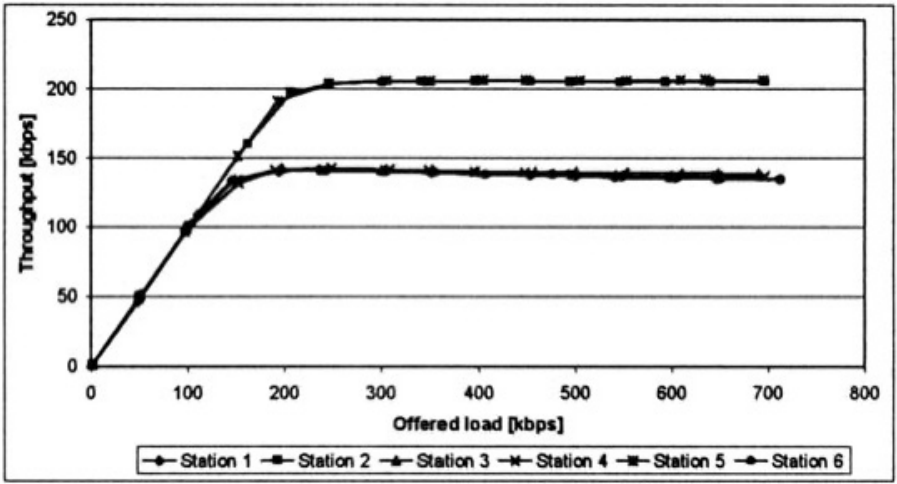

Fig. 4. Throughput versus offered load for Scenario B, RTS/CTS mechanism always enabled

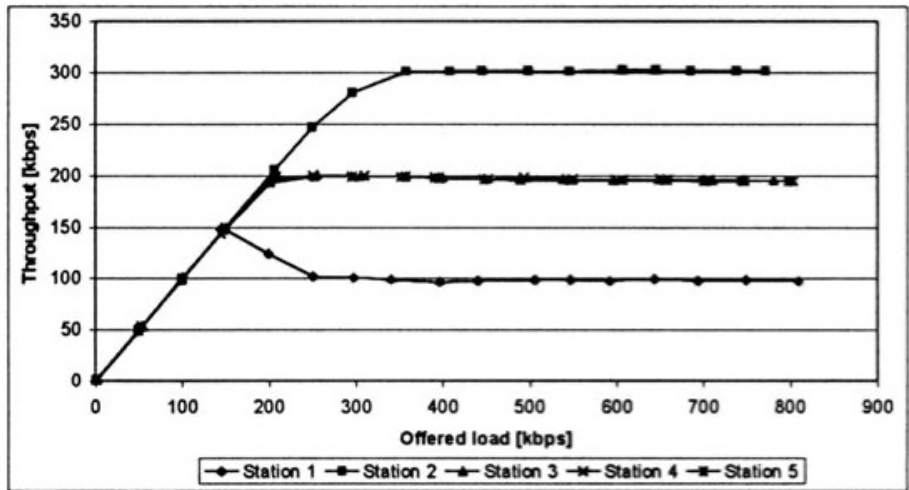

Fig. 5. Throughput versus offered load for Scenario C, RTS/CTS mechanism always enabled

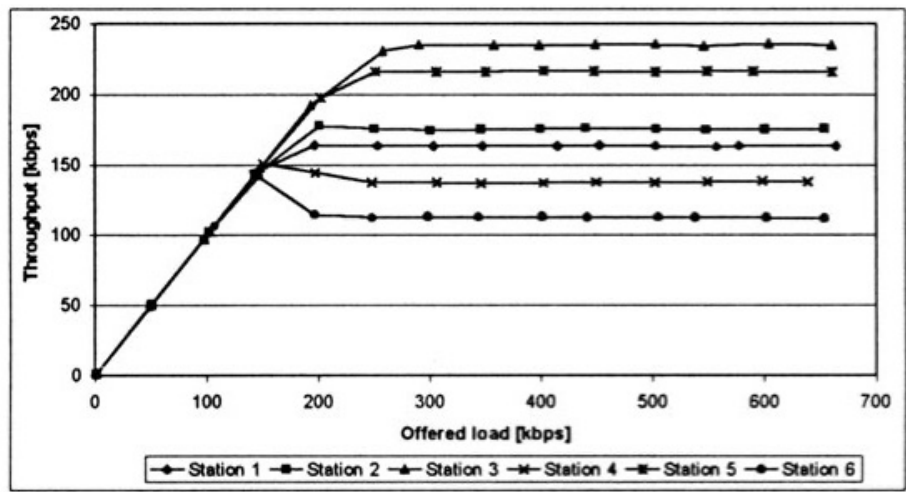

Fig. 6. Throughput versus offered load for Scenario D, RTS/CTS mechanism always enabled 


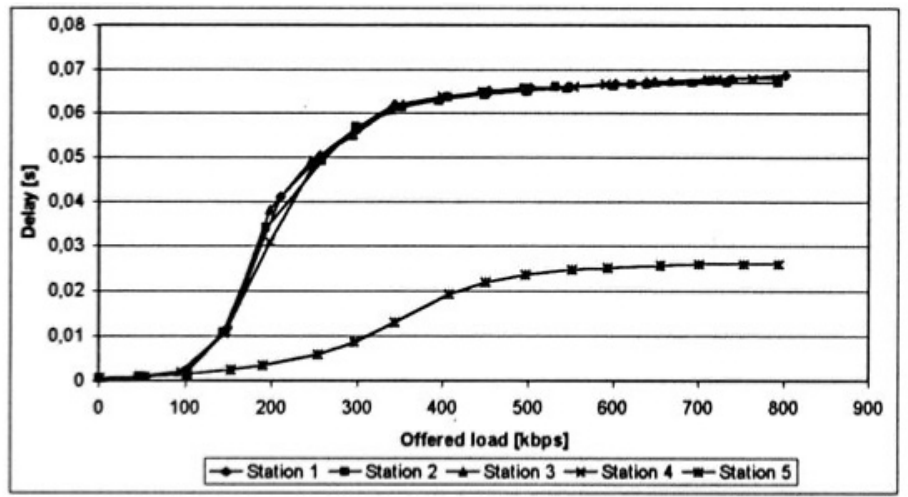

Fìg. 7. Mean packet delay versus offered load for Scenario A, RTS/CTS mechanism enabled

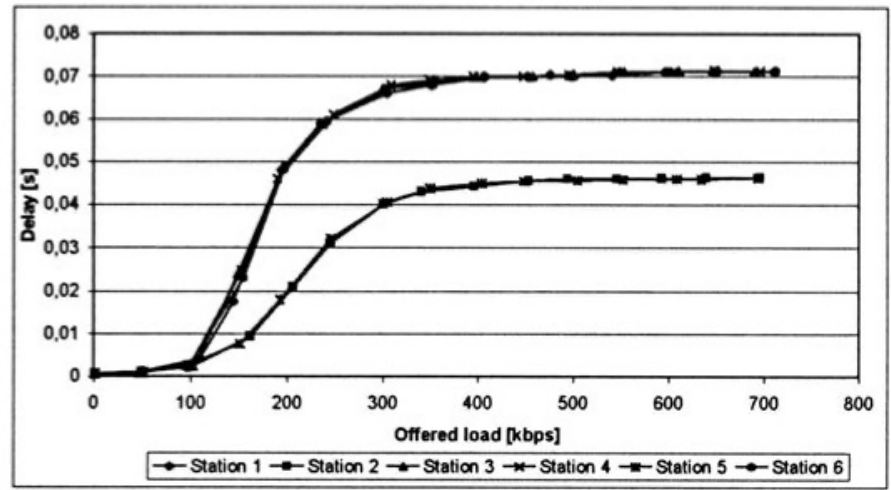

Fig. 8. Mean packet delay versus offered load for Scenario B, RTS/CTS mechanism enabled

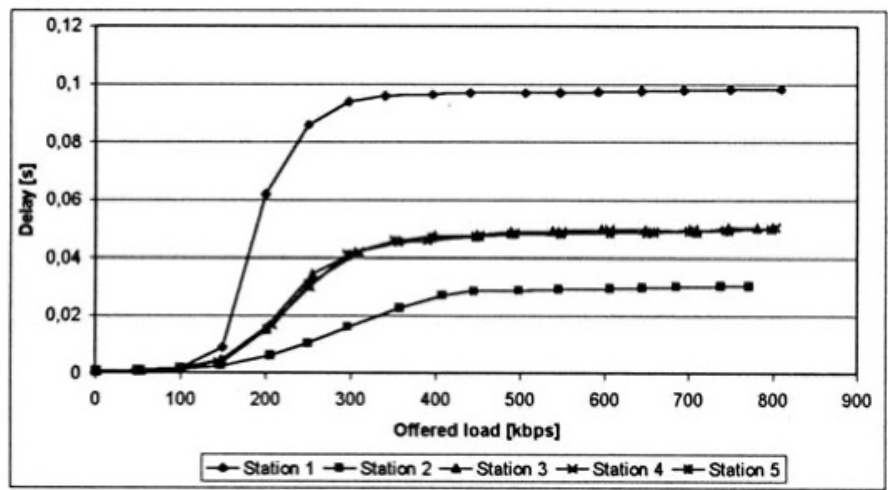

Fig. 9. Mean packet delay versus offered load for Scenario C. RTS/CTS mechanism enabled 


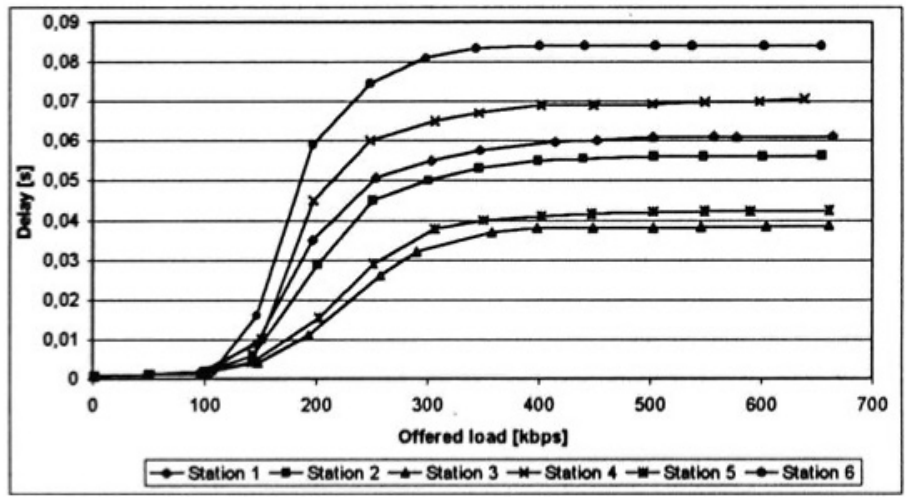

Fig. 10. Mean packet delay versus offered load for Scenario D, RTS/CTS mechanism enabled

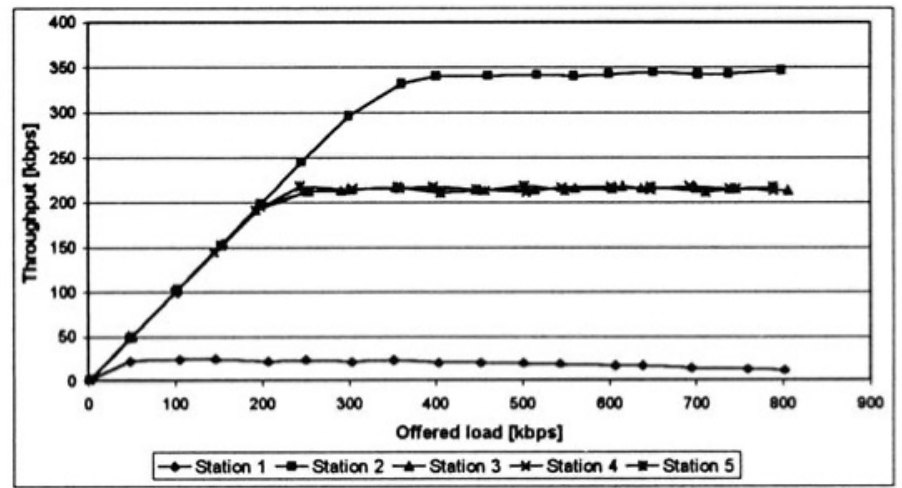

Fig. 11. Throughput versus offered load for Scenario C, RTS/CTS mechanism always disabled

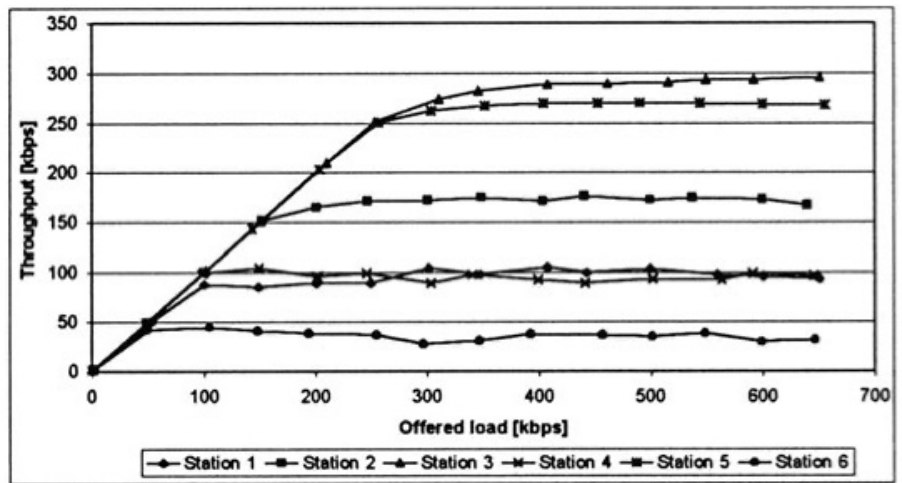

Fig. 12. Throughput versus offered load for Scenario D, RTS/CTS mechanism disabled 


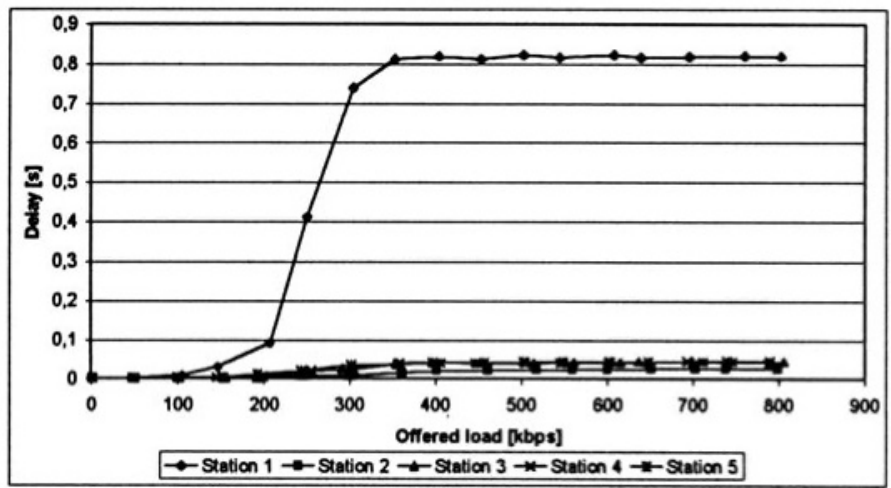

Fig. 13. Mean packet deloy versus offered load for Scenario C, RTS/CTS mechanism disabled

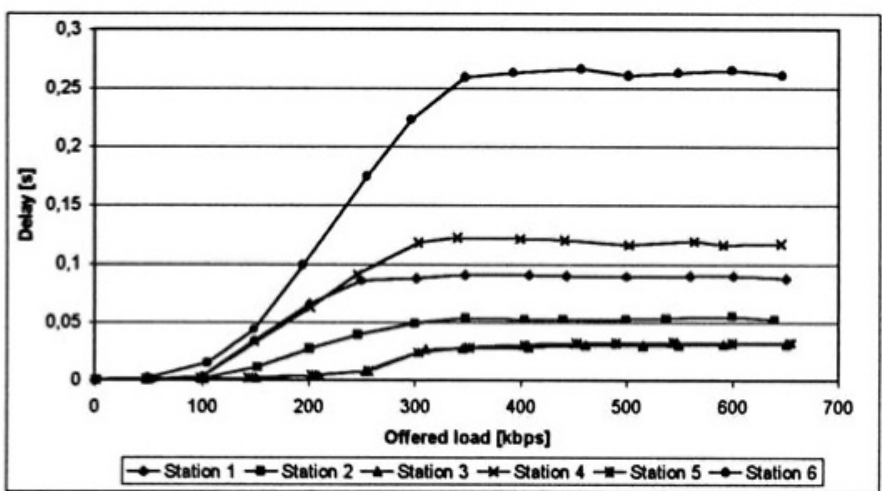

Fig. 14. Mean packet delay versus offered load for Scenario D. RTS/CTS mechanism disabled

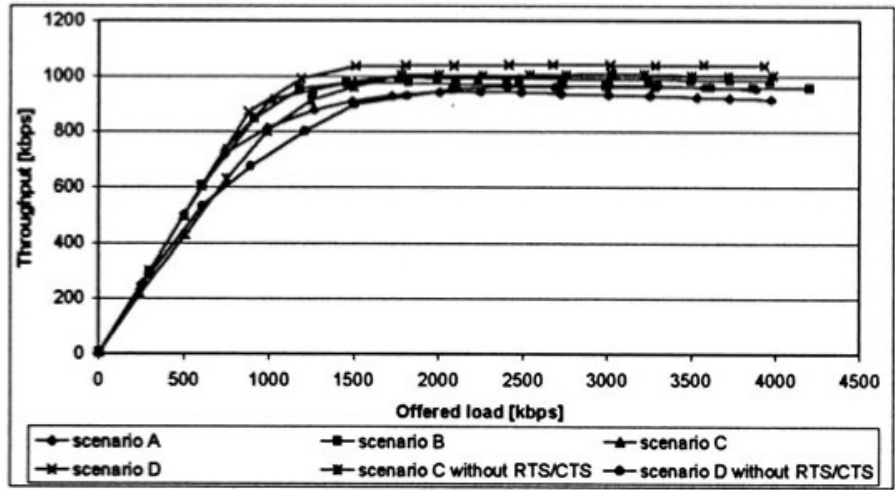

Fig. 15. Throughput versus offered load for all 6 scenarios 


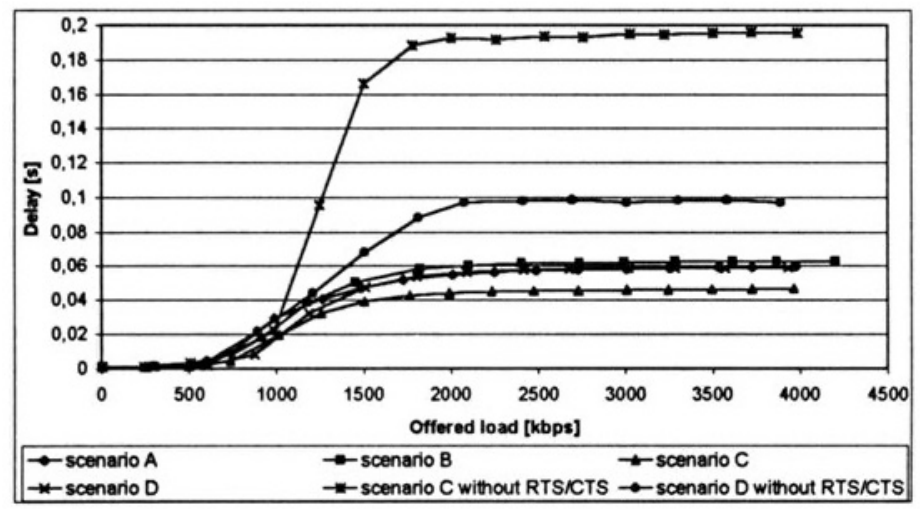

Fig. 16. Mean packet delay versus offered load for all 6 scenarios

\section{DISCUSSION}

The presented work describes a simulation analysis of hidden station influence on the IEEE 802.11 network efficiency. Four different hidden station scenarios were investigated. The throughput (overall and obtained for every station) and the mean packet delay as a function of the offered load were studied. They allow us to determine the usefulness of RTS/CTS mechanism usage. The obtained results allow us to draw some general conclusions about the IEEE 802.11 network efficiency in the presence of hidden stations:

- The presence of hidden stations brings significant network performance degradation.

- A growth of the offered load above the nominal capacity of the network does not brings the degradation of the realized throughput like in some others wireless networks (IEEE 802.11 MAC protocol is more stable).

- The presence of hidden station causes unfaimess in access to the medium - stations located in the center have larger transmission privilege.

- Hidden stations frequently loose medium access competition for larger values of the offered load and, therefore, reach smaller throughputs and considerable higher delays.

- The RTS/CTS mechanism improves the faimess of network operation. It brings the growth of the realized throughput (even of hundreds percents) and reduction of the mean packet delay (up to many times) for hidden stations.

- The positive influence of the RTS/CTS mechanism usage grows with the number of stations (especially hidden), offered load and length of transmitted packets. 


\section{CONCLUSIONS}

The presented analysis allow us to determine the efficiency of an IEEE 802.11 network in the presence of hidden stations. The presented study demonstrates the reasonableness of RTS/CTS mechanism usage. The obtained efficiency is, of course, dependent on many factors as the number of contending stations, the type of traffic, the offered load, the number of hidden stations, etc. The obtained results shows that it is better to use the RTS/CTS mechanism especially when the hidden stations location is unknown.

\section{REFERENCES}

[1] BHARGHAVAN V., Performance Analysis of a Medium Access Protocol for Wireless Packet Networks. IEEE Performance and Dependability Symposium '98, Raleigh, NC. August 1998.

[2] FULLMER C. L., GARCIA-LUNA ACEVES J. J., Solution to Hidden Terminal Problems in Wireless Networks. ACM SIGCOMM Conference on Communications Architectures, Protocols and Architectures, Cannes, France, September 1997

[3] HAAS Z. J., On the performance of a medium access control scheme for the reconfigurable wireless networks. IEEE Milcom'97, Nov. 1997

[4] IEEE 802.11 Standard for Wireless LAN: Medium Access Control (MAC) and Physical Layer (PHY) Specification. New York, IEEE Inc. 1997

[5] KARN P., MACA - A new channel access method for packet radio. in ARRL/CRRL Amateur Radio $9^{\text {th }}$ Computer Networking Conference 1990

[6] NATKANIEC M., PACH A. R: Simulation Analysis of Multimedia Streams Transmission in IEEE 802.11 Networks. - ISWC'99 IEEE International Symposium on Wireless Communications, June 1999, Victoria, Canada

[7] SOUSA E., SILVESTER J. A., Spreading code protocols for distributed spread-spectrum packet radio networks. IEEE Trans. Commun., vol. 36 no. 3, Mar. 1988

[8] TOBAGI F. A., KLEINROCK L., Packet switching in radio channels: Part I - carrier sense multiple-access modes and their throughput delay characteristics. IEEE Trans. Commun. vol. COM-23, no. 12, 1975

[9] TOBAGI F. A., KLEINROCK L., Packet switching in radio channels: Part II - the hidden terminal problem in carrier sense multiple-access modes and the busy-tone solution. IEEE Trans. Commun. vol. COM-23, no. 12, 1975

[10] WEINMILLER J., WOESNER H., EBERT J-P.., WOLISZ A., Analysing the RTS/CTS Mechanism in the DFWMAC Media Access Protocol for Wireless LANs, IFIP TC6 Workshop Personal Wireless Communications, April 1995, Prague Czech Republic

[11] WOŹNIAK J., NOWICKI K., How the Usage of RTS/CTS and HNA Mechanisms Can Improve the Fairness of Operation of IEEE 802.11 and HIPERLAN Networks In the Presence of Hidden Stations?- $6^{\text {th }}$ Polish Teletraffic Symposium 22-23 April 1999 Szklarska Poręba

[12] WU C., LI V. O. K., Receiver-initiated busy-tone multiple access in packet radio networks. ACM SIGCOMM 87 Workshop: Frontiers in Computer Communications Technology, Stowe, VT, USA 11-13 Aug. 1987 